\title{
Two Cases of Foveal Hypoplasia with Different Etiologies
}

\author{
Abdullah Ozkaya, ${ }^{1}$ Hatice Nur Tarakcioglu ${ }^{2}$ \\ 1Department of Ophthalmology, Beyoglu Eye Training and Research Hospital, Istanbul, Turkey \\ ${ }^{2}$ Department of Ophthalmology, Bakirkoy Dr. Sadi Konuk Training and Research Hospital, Istanbul, Turkey
}

\begin{abstract}
In this report the optical coherence tomography (OCT) findings of 2 patients with foveal hypoplasia are presented. One patient had oculocutaneous albinism, which is a frequent cause of foveal hypoplasia, and had decreased visual acuity in both eyes. The second patient did not have an associated disease. The clinical and OCT findings of both patients are summarized.
\end{abstract}

Keywords: Albinism, fovea, hypoplasia.

\section{Introduction}

Foveal hypoplasia may be more accurately defined as fovea plana, which is described as the absence of a foveal pit and the presence of inner retinal layers at the central macula (I5). In clinical practice, foveal hypoplasia may be associated with albinism, retinopathy of prematurity, aniridia, nanophthalmos, incontinentia pigmenti, and less often, may be an isolated entity (I-5). Presently described are the results of optical coherence tomography (OCT) scans and infrared photographs of 2 cases of foveal hypoplasia, I of which was associated with albinism, while the other was an isolated case.

\section{Case Report}

Case I: A 6-year-old girl was presented in May 2014 with decreased visual acuity and nystagmus. She had a history of oculocutaneous albinism. Her family history was not notable. On physical examination, it was noted that the patient had blonde hair and very pale skin. When asked, her parents reported that the patient was not able to suntan. An ocular examination revealed a best corrected visual acuity (BCVA) of 0.4 decimals in both eyes. Examination of the anterior segment revealed a diffuse iris transillumination defect in both eyes. Both eyes also displayed nystagmus. Fundus examination revealed a faint foveal reflex, macular hypoplasia, diffuse retinal pigment epithelial atrophy, and prominent choroidal vessels in both eyes (Fig. I). An OCT scan showed the absence of a foveal pit, the presence of inner retinal layers, and an intact outer segment ellipsoid zone in both eyes (Fig. I). The patient was diagnosed with foveal hypoplasia secondary to albinism and periodic follow-up visits were recommended.

Case 2: A 9-year-old girl was presented in April 2015 with the complaint of decreased visual acuity. Her history and family history were not remarkable. A physical examination of the patient revealed no prominent abnormality. An ocular examination indicated a BCVA of 0.4 decimals in the right eye and 0.3 decimals in the left eye. Anterior segment examination was normal in both eyes. Nystagmus was not detected. Fundus examination revealed the absence of a foveal reflex and macular hypoplasia in both eyes (Fig. 2). An OCT

Address for correspondence: Abdullah Ozkaya, MD. Beyoglu Goz Egitim ve Arastirma Hastanesi, Bereketzade Cami Sokak, 34421 Beyoglu, Istanbul, Turkey

Phone: +90 2122515900 E-mail: abdozkaya@gmail.com

Submitted Date: November 07, 2017 Accepted Date: November 11, 2017 Available Online Date: November 27, 2017 ${ }^{\circ}$ Copyright 2017 by Beyoglu Eye Training and Research Hospital - Available online at www.beyoglueye.com 

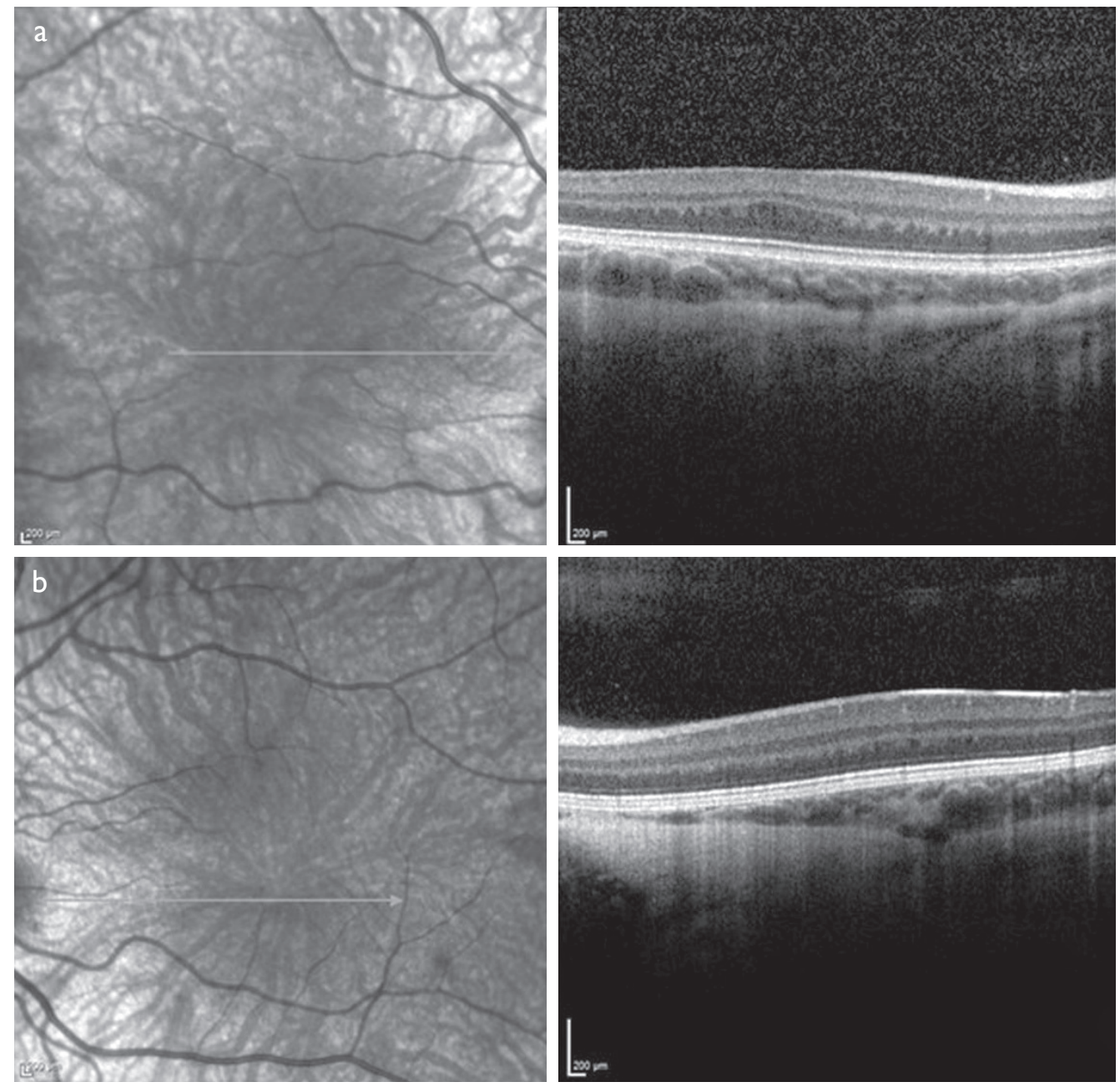

Figure I. Patient I (a) The infrared image and optical coherence tomography (OCT) scan of the right eye; (b) The infrared image and OCT scan of the right eye. Both infrared images show prominent choroidal vessels. Both images show the absence of a foveal pit.

scan showed the absence of a foveal pit, the presence of inner retinal layers, and an intact outer segment ellipsoid zone in both eyes (Fig. 2). The patient was diagnosed as having isolated foveal hypoplasia secondary albinism and periodic follow-up visits were recommended.

\section{Discussion}

The fovea is responsible for high central visual acuity and color vision (3). The development of the fovea was found to be associated with the development of visual pathways and the gray matter of the occipital cortex (3). It is immature at birth; foveal maturation occurs postnatally along with the centrifugal migration of the inner retinal layers and centripetal migration of cone cells (3). Foveal hypoplasia is usually associated with previously described clinical entities (I-5). Oculocutaneous albinism is a common disease characterized by systemic features such as blonde hair, pale skin, and the inability to suntan, as well as ocular features such as decreased visual acuity, photophobia, transillumination of the iris, nystagmus, and a hypopigmented fundus (I, 2). McCafferty et al. (2) evaluated the foveal morphology of I4 patients with albinism. They reported that the BCVA of the patients varied from 20/20 to 20/100 and that a better BCVA was associated with a preserved foveal avascular zone, annular reflex of the macula, stereo acuity, and macular melanin. Only 3 of the patients were found to have a faint foveal pit, whereas the others lacked a foveal pit. Our first case also displayed most of the typical systemic and ocular findings of oculocutaneous albinism. Maldonado et al. (3) reported that most of the premature infants studied demonstrated the presence of inner retinal layers between the age of $3 \mathrm{I}$ and 33 weeks and concluded that human foveal development continued after premature birth. Basilius et al. (4) demonstrated structural abnormalities of the macula in 5 incontinentia pigmenti patients. Along with the other systemic and ocular features of incontinentia pigmenti, 3 eyes out of 10 eyes in 5 patients 

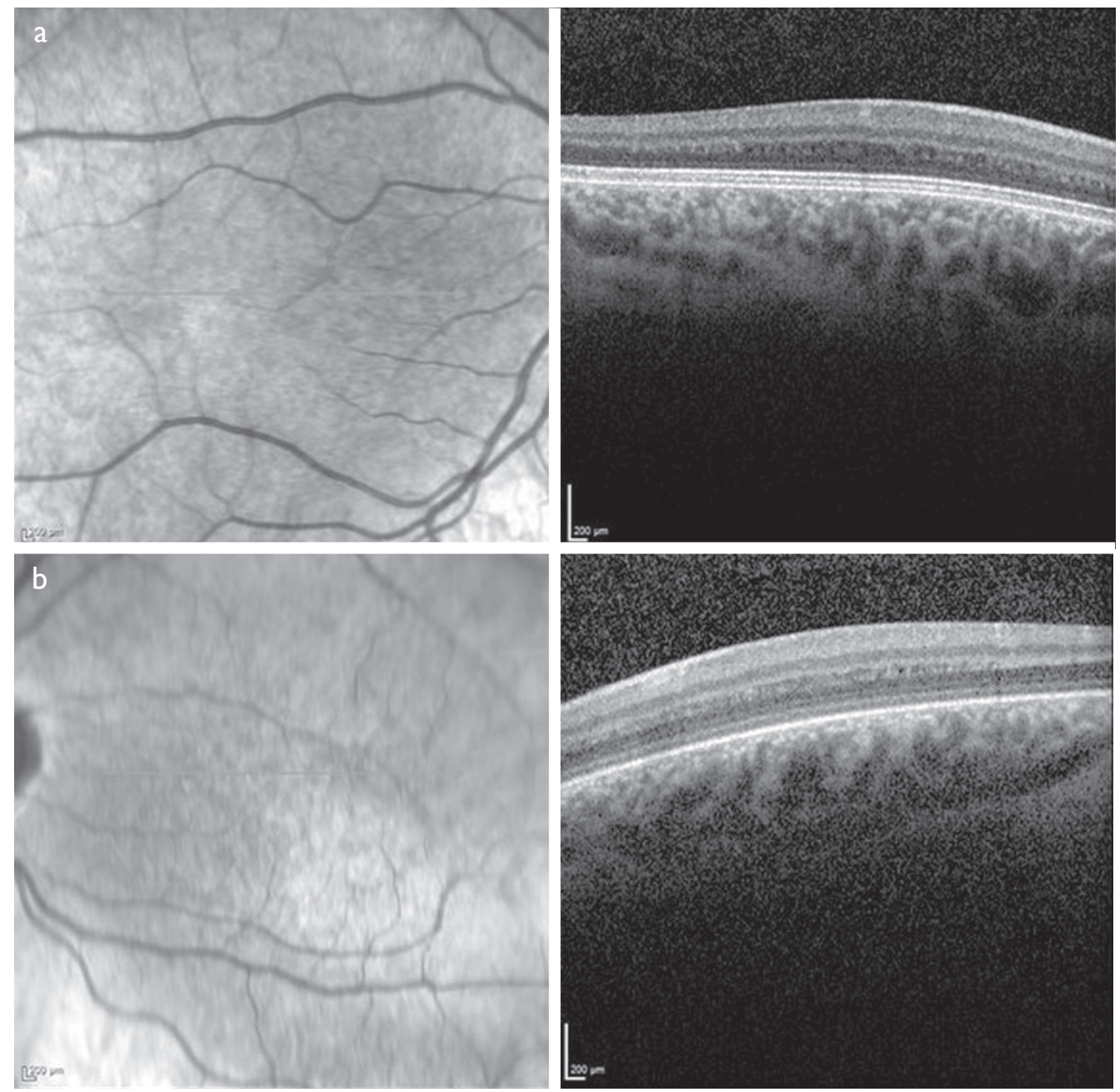

Figure 2. Patient 2 (a) The infrared image and optical coherence tomography scan of the right eye; (b) The infrared image and OCT scan of the right eye. Both images illustrate the absence of a foveal pit.

showed the absence of a foveal pit in their study. Marmor et al. (5) evaluated the visual of significance of a foveal pit in 4 patients who lacked a foveal pit. They suggested the term foveal plana instead of foveal hypoplasia for patients with a good visual acuity despite absence of a foveal pit. Both of our patients demonstrated the absence of a foveal pit with a fairly good visual acuity. The outer segment of retinal layers was healthy in both patients, and the outer segment ellipsoid zone was normal in all 4 eyes of the 2 patients. Perhaps these two patients should be said to have foveal plana instead of foveal hypoplasia.

In conclusion, foveal hypoplasia can be associated with several systemic and ocular diseases that might affect visual acuity. However, it may also be a benign isolated finding of an ocular examination when other causes are excluded.

\section{Disclosures}

Peer-review: Externally peer-reviewed.

Conflict of Interest: None declared.
Authorship Contributions: Involved in design and conduct of the study (AO, HNT); preparation and review of the study (AO, HNT); data collection (AO, HNT).

\section{References}

I. Pal SS, Gella L, Sharma T, Raman R. Spectral domain optical coherence tomography and microperimetry in foveal hypoplasia. Indian J Ophthalmol 201 1;59:503-5. [CrossRef]

2. McCafferty BK, Wilk MA, McAllister JT, Stepien KE, Dubis AM, Brilliant $\mathrm{MH}$, et al. Clinical Insights Into Foveal Morphology in Albinism. J Pediatr Ophthalmol Strabismus 2015;52:167-72.

3. Maldonado RS, O'Connell RV, Sarin N, Freedman SF, Wallace DK, Cotten CM, et al. Dynamics of human foveal development after premature birth. Ophthalmology 201।;1 18:2315-25.

4. Basilius J, Young MP, Michaelis TC, Hobbs R, Jenkins G, Hartnett ME. Structural Abnormalities of the Inner Macula in Incontinentia Pigmenti. JAMA Ophthalmol 2015;133:1067-72. [CrossRef]

5. Marmor MF, Choi SS, Zawadzki RJ, Werner JS. Visual insignificance of the foveal pit: reassessment of foveal hypoplasia as fovea plana. Arch Ophthalmol 2008;126:907-13. [CrossRef] 